\title{
Sex Assessment by Morphological Analysis of Palatal Rugae Patterns in a South Indian Adult Population
}

This article was published in the following Dove Press journal:

Clinical, Cosmetic and Investigational Dentistry

\author{
Komal Smriti ${ }^{1}$ \\ Raj Gupta ${ }^{2}$ \\ Kalyana C Pentapati iD ${ }^{3}$ \\ Anupam Singh ${ }^{4}$ \\ Ira Kapoor ${ }^{2}$ \\ Ravindranath Vineetha' \\ Srikanth Gadicherla ${ }^{4}$ \\ 'Department of Oral Medicine and \\ Radiology, Manipal College of Dental \\ Sciences, Manipal, Manipal Academy of \\ Higher Education, Manipal, India; \\ ${ }^{2}$ Manipal College of Dental Sciences, \\ Manipal, Manipal Academy of Higher \\ Education, Manipal, India; ${ }^{3}$ Department of \\ Public Health Dentistry, Manipal College \\ of Dental Sciences, Manipal, Manipal \\ Academy of Higher Education, Manipal, \\ India; ${ }^{4}$ Department of Oral and \\ Maxillofacial Surgery, Manipal College of \\ Dental Sciences, Manipal, Manipal \\ Academy of Higher Education, Manipal, \\ India
}

Background: Palatal rugae are asymmetric and irregular ridges on the anterior palatal region, which have proven to be useful in the field of forensic dentistry. This study aims to use morphological analysis of palatal rugae patterns to assess sex in a South Indian adult population. Materials and Methods: This cross-sectional study was undertaken to evaluate a total of 300 maxillary dental casts (150 males and 150 females). The rugae patterns were marked by one observer and classified as per Thomas and Kotze criteria by another observer. The rugae patterns were classified based on the total number of rugae, shape, predominant direction of rugae, and unification of rugae.

Results: The palatal rugae in female participants showed a higher incidence of circular rugae and backward directed rugae, while male participants showed more forward-directed rugae. The logistic regression analysis showed a significant association of with circular $(\mathrm{OR}=1.48 ; 95 \% \mathrm{CI}=1.02-2.13)$, forward $(\mathrm{OR}=0.87 ; 95 \% \mathrm{CI}=0.78-0.97)$ and backward $(\mathrm{OR}=1.23 ; 95 \% \mathrm{CI}=1.08-1.41)$ palatal rugae with sex.

Conclusion: Certain palatal rugae patterns can be used for the assessment of sex with limited accuracy.

Keywords: palatal rugae, sex, identification, palatoscopy, forensic, dentistry

\section{Introduction}

Forensic dentistry is a field of dentistry that deals with medico-legal identification using dental findings. ${ }^{1}$ Human identification post-death involves proper evaluation and correlation of dental records, DNA testing, and fingerprinting. Palatal rugae (PR) are one such dental records that are asymmetric and irregular ridges present on the palatal mucosa's anterior portion on either side of the median palatine raphe. The pattern and position of these rugae have led to their study known as palatoscopy. In recent years, palatoscopy or rugoscopy has gained importance and emerged as a suitable technique in establishing identity post-mortem when dental records cannot be used, like in edentulous individuals or when there is a decomposition of the body. It was first introduced by a Spanish investigator named Troban Hermoso (1932). Lysell proposed the first organized classification of PR. ${ }^{2}$ Later the modified and most acceptable classification was put forward by Thomas and Kotze.

Besides rugae patterns being unique to individuals, studies have shown that extraction and orthodontic treatment show no apparent effects on rugae allowing them to be used as stable reference points in analysis and identification. ${ }^{3}$ Furthermore, the rugae have proven to show less pronounced changes than the generalized body parts in charring and decomposition cases.
Correspondence: Kalyana C Pentapati Department of Public Health Dentistry, Manipal College of Dental Sciences, Manipal, Manipal Academy of Higher Education, Manipal, Karnataka, India Email drkalyan8I@gmail.com
Clinical, Cosmetic and Investigational Dentistry 2021:13 77-8I 
A considerable amount of literature exists on diverse topics like genetic predisposition, ${ }^{2}$ the prevalence of various morphological patterns, ${ }^{4-9}$ and differences in PR's morphology concerning sex. ${ }^{2,10-17}$ To date, there has been little agreement on whether sex can be predicted with the help of PR patterns ${ }^{11,18}$ and most of the studies recommended the use of PR along with other methods for the identification of sex. With this background, we aimed to use the morphological analysis of palatal rugae patterns to assess sex in a South Indian adult population.

\section{Materials and Methods}

We conducted a retrospective cross-sectional study on the casts of patients who required Orthodontic treatment planning and prosthesis fabrication. Based on the findings from the previous study, ${ }^{14}$ an effect size of 0.4 was estimated. The sample size was estimated to be 216 (alpha 5\% and 90\% power), rounded to 220 . A total of 300 maxillary casts (150 males and 150 females) were sought. The institutional ethics committee of Kasturba hospital and Kasturba medical college, Manipal, approved the study protocol (IEC 39/2019). This study was conducted as per the declaration of Helsinki.

Criteria for selecting the casts were as follows: age range of 18-40 years, Indian origin, and those willing to participate. Casts with palatal pathologies, edentulous, history of any previous trauma or palatal surgeries or cleft palate, and those undergoing fixed orthodontic treatment were excluded $(n=48)$.

The rugae patterns were marked with a black color marker $^{19}$ under adequate illumination by one observer. The variables included for the assessment were the total number of rugae, shape, predominant direction of rugae, and rugae's unification. The full length of the rugae was classified as primary rugae (more than $5 \mathrm{~mm}$ ) or secondary rugae ( 3 to $5 \mathrm{~mm}$ ). According to each rugae's shape, it can be classified as straight, curved, wavy, and circular. ${ }^{2}$ The straight rugae run continuously from origin to the insertion point in undeviated fashion. The crescent-shaped rugae with a gentle bend are classified as a curved type. The serpentine-shaped rugae were categorized as wavy, and the rugae, which formed a ring by joining one end to the other, are termed circular rugae. If the rugae split to form two arms, it is termed as "unification" or "branches." Unification is the term given according to Thomas and Kotze classification to the rugae, which are either joined at their origin or the termination. ${ }^{19}$ If the rugae have a common origin, branches later are called diverging, whereas two different rugae join at termination is named converging. The rugae's direction is determined by the angle formed by the origin and terminal end of rugae concerning mid palatine raphae. The rugae with positive angles or acute are termed forward rugae. The rugae with a negative or obtuse angle are called backward rugae, and the rugae with zero angle are termed perpendicular rugae (Figure 1). One observer made the identification, classification and recording the number of the rugae. Intra-observer reliability was assessed using intra-class correlation coefficient which ranged from (0.87 to 0.93$)$.

\section{Statistical Analysis}

All analyses were performed using SPSS 20 (IBM Corp. Released in 2011. IBM SPSS Statistics for Windows, Version 20.0. Armonk, NY: IBM Corp). Normality was assessed using the Kolmogorov Smirnov test. The data was found non-normal and non-parametric tests were conducted. Mann-Whitney $U t$-test was performed to evaluate the sex differences with various PR patterns. Binary logistic regression analysis was performed to assess the sex with different PR patterns. Hosmer Lemeshow goodness of fit was used to evaluate the model fit. A P-value of $<0.05$ was considered statistically significant.

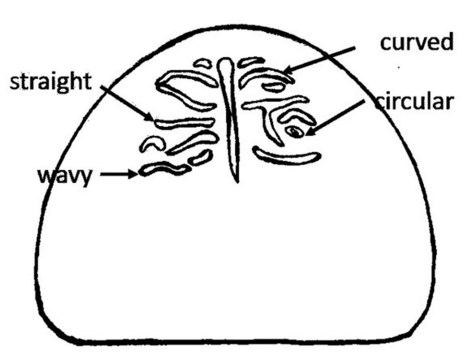

Types of rugae based on shapes

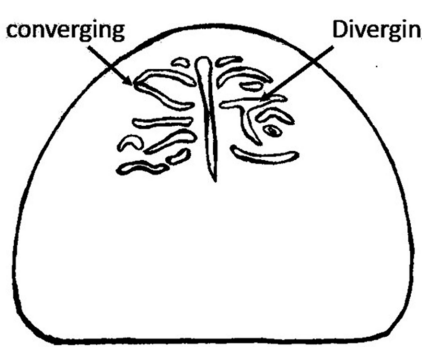

Types of rugae based on unification

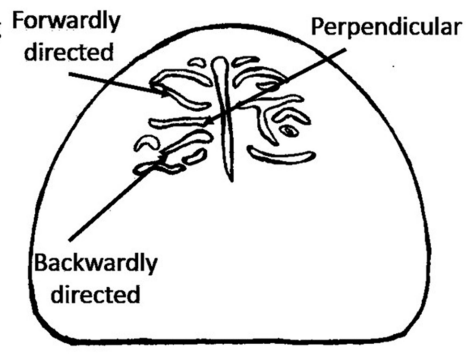

Types of rugae based on direction

Figure I Schematic description of various palatal rugae patterns used for assessment in this study. 


\section{Results}

A total of 252 casts were evaluated, out of which 132 were male. Table 1 shows the distribution of PR patterns concerning sex. There was a significant difference in the distribution of circular and backward PR patterns between males and females $(\mathrm{P}=0.05$ and 0.01$)$, respectively (Table 1$)$. The results, as shown in Table 2, indicated that females had a higher incidence of circular rugae $(\mathrm{p}=0.049)$ and backward directed rugae $(\mathrm{p}=0.001)$, while males showed more forward-directed rugae $(p=0.006)$ (Table 2). No other significant difference in the patterns was evident between males and females.

We performed three independent binary logistic regression analyses to assess the sex using the variables that were significant in bivariate analysis. There was significant association of sex with circular $(\mathrm{OR}=1.48 ; 95 \% \mathrm{CI}=1.02-2.13)$, forward $(\mathrm{OR}=0.87 ; 95 \% \mathrm{CI}=0.78-0.97)$ and backward $(\mathrm{OR}=1.23$; $95 \% \mathrm{CI}=1.08-1.41$ ) palatal rugae patterns (Table 3 ).

\section{Discussion}

PR are permanent, stable, and unique characteristics that can establish identity in the absence of antemortem records. ${ }^{20}$ Literature has shown diverse applications and facts about the PR in forensic dentistry. This individuality and accuracy of patterns even extend to monozygous ${ }^{20}$ and dizygous twins. Also, forensic identification with the help of PR seems promising in the absence of fingerprints. Previous studies evaluating the stability of PR along the entire growth period of an individual were inconsistent. Hauser et al observed that the number of PR differs in different ethnic races and between sex and increases slightly during adolescence and distinctly above the age of 35 years. $^{21}$ Lysell et al proposed that the number of PR decreases in an individual from 23 years onwards, but PR's characteristic pattern does not change

Table I Distribution of Palatal Rugae in Males and Females

\begin{tabular}{|l|r|r|r|}
\hline & \multicolumn{1}{|c|}{ Male } & Female & P-value \\
\hline Curved & 118 & 107 & 0.95 \\
Wavy & 125 & 108 & 0.16 \\
Straight & 98 & 89 & 0.99 \\
Circular & 25 & 35 & $0.05^{*}$ \\
Forward & 129 & 118 & $>0.99$ \\
Backward & 108 & 111 & $0.01^{*}$ \\
Perpendicular & 47 & 41 & 0.81 \\
Converging & 131 & 117 & 0.35 \\
Diverging & 130 & 117 & 0.67 \\
\hline
\end{tabular}

Note: *Indicates $\mathrm{P} \leq 0.05$
Table 2 Comparison of the Mean Number of PR Between Males and Females

\begin{tabular}{|c|c|c|c|}
\hline \multirow[t]{3}{*}{ PR Patterns } & \multicolumn{2}{|c|}{ Sex } & \multirow[t]{3}{*}{ P-value } \\
\hline & Male & Female & \\
\hline & Mean \pm SD & Mean \pm SD & \\
\hline \multicolumn{4}{|l|}{ Shape } \\
\hline Curved & $3.17 \pm 2.32$ & $3.47 \pm 2.25$ & 0.221 \\
\hline Wavy & $3.98 \pm 2.46$ & $3.56 \pm 2.15$ & 0.232 \\
\hline Straight & $1.5 \pm 1.46$ & $1.39 \pm 1.36$ & 0.752 \\
\hline Circular & $0.26 \pm 0.59$ & $0.45 \pm 0.83$ & $0.049 *$ \\
\hline Total & $8.91 \pm 1.95$ & $8.87 \pm 1.83$ & 0.979 \\
\hline \multicolumn{4}{|l|}{ Direction } \\
\hline Forward & $5.77 \pm 2.28$ & $5.06 \pm 2.2$ & $0.006 *$ \\
\hline Backward & $2.28 \pm 2.02$ & $3.05 \pm 1.8$ & $<0.001 *$ \\
\hline Perpendicular & $0.82 \pm 1.53$ & $0.72 \pm 1.22$ & 0.847 \\
\hline \multicolumn{4}{|l|}{ Unification } \\
\hline Converging & $4.53 \pm 1.71$ & $4.52 \pm 1.97$ & 0.970 \\
\hline Diverging & $2.17 \pm 1.11$ & $2.15 \pm 1.30$ & 0.408 \\
\hline
\end{tabular}

Note: *Indicates $\mathrm{P} \leq 0.05$.

Abbreviations: PR, palatal rugae; SD, standard deviation.

Table 3 Assessment of Sex Using Different Palatal Rugae Patterns

\begin{tabular}{|l|l|l|l|l|}
\hline $\begin{array}{l}\text { Palatal Rugae } \\
\text { Pattern }\end{array}$ & P-value & OR & 95\% Cl & $\begin{array}{l}\text { Percentage } \\
\text { Correct }\end{array}$ \\
\hline Circular & $0.038^{*}$ & 1.48 & $1.02-2.13$ & $56.3 \%$ \\
Forward & $0.013^{*}$ & 0.87 & $0.78-0.97$ & $55.2 \%$ \\
Backward & $0.002^{*}$ & 1.23 & $1.08-1.41$ & $60.3 \%$ \\
\hline
\end{tabular}

Note: *Indicates $\mathrm{P} \leq 0.05$

Abbreviations: $\mathrm{OR}$, odds ratio; $\mathrm{Cl}$, confidence interval.

with time. ${ }^{2}$ Few researchers stated that PR remains unchanged since the 12 th week of intrauterine life. ${ }^{8}$

Given the potential applications of palatoscopy in the field of forensic dentistry, our study evaluated the possibility of predicting sex using the PR patterns. Wavy and curved PR were the most frequently reported in our study. This finding broadly supports the results of earlier reports. $4,6,7,9-11,13-15,18,22$

The current study found that only the number of circular rugae was significantly higher in females than males, similar to an earlier report. ${ }^{7}$ However, the findings of our study do not support the results of previous studies. ${ }^{23}$ Studies have reported that males' most common pattern was a wavy pattern ${ }^{9,24,25}$ and in females was a curved pattern. 9,14 The wavy pattern followed by a straight pattern was predominantly seen in females, while the curved pattern was seen most commonly in males. ${ }^{11,25,26}$

We observed no significant differences in convergence and divergence patterns in males and females, which 
accords with earlier studies. ${ }^{7,14}$ However, a previous study showed that males had more converging rugae, while females had more diverging rugae. ${ }^{11,26}$ It was also seen that males had more forwardly directed rugae than females, similar to an earlier report. ${ }^{12}$ Pereira et $\mathrm{al}^{11}$ reported that both forward and backward were common in males, while perpendicular patterns were common in females, while Dwivedi and Nagarajappa reported that backwardly directed rugae were common in males. ${ }^{24}$

In agreement with our study's findings, previous studies have demonstrated no significant difference between males and females. ${ }^{4,5}$ This finding was contrary to an earlier study that reported a higher number of rugae in males than females. ${ }^{6}$

Along with the evaluation of PR patterns and sex differences in PR's distribution, we aimed to evaluate the role of $\mathrm{PR}$ in the assessment of sex using the logistic regression analysis. Direct comparison of our study with the existing studies may not be feasible as only a limited number of studies exist. Only a few significant PR patterns could be used to predict sex. Similar accuracy $(61 \%)$ was reported among Iranian school children, ${ }^{18}$ and but higher values (90\% and $99.2 \%)$ were also reported. ${ }^{11,23}$ However, studies have reported no significant difference in the PR patterns concerning $\operatorname{sex}^{4,27}$ while a significant difference in the number of rugae between transgender and males or females. $^{12}$

PR's are stable, reliable, easy to visualize, and simple to record with minimal cost. Due to the numerous advantages, it has applications in forensic identification. Few limitations exist in this study. Participants were only south Indians and were selected from the patient pool.

\section{Conclusion}

Within this study's limits, we can conclude that PR has a possible role in identifying the sex but with limited accuracy. It may be considered along with other reliable forensic tools as a supplementary aid in identifying sex. Further work is needed in diverse populations and ethnic groups to explore PR's potential in forensic dentistry. In future investigations, a combination of other dental parameters with PR can identify sex with a high level of accuracy.

\section{Abbreviations}

$\mathrm{PR}$, palatal rugae; OR, odds ratio; $\mathrm{CI}$, confidence interval.

\section{Ethics Approval and Consent to Participate}

This study was conducted as per the Declaration of Helsinki. The institutional ethics committee of Kasturba Medical College and Kasturba Hospital, Manipal Academy of Higher Education, Manipal approved the study protocol (IEC 39/ 2019). Studies involving research in humans initiated by researchers of various associated institutions under the aegis of Manipal Academy of Higher Education, Manipal, come under the Kasturba Medical College and Kasturba Hospital's purview ethics committee. Informed consent was obtained from all the participants.

\section{Disclosure}

The authors report no conflicts of interest for this work.

\section{References}

1. Shamim T, Ipe Varughese V, Shameena PM, Sudha S. Forensic odontology - a new perspective. Med Legal Update. 2006;6(1):1-4.

2. Lysell L. Plicae palatinae transversae and papilla incisiva in man; a morphologic and genetic study. Acta Odontol Scand. 1955;13 (Suppl. 18):5-137. doi:10.1016/0002-9416(55)90193-7

3. Peavy DC, Kendrick GS. The effects of tooth movement on the palatine rugae. J Prosthet Dent. 1967;18(6):536-542. doi:10.1016/ 0022-3913(67)90219-3

4. Kapali S, Townsend G, Richards L, Parish T. Palatal rugae patterns in Australian Aborigines and Caucasians. Aust Dent J. 1997;42 (2):129-133. doi:10.1111/j.1834-7819.1997.tb00110.x

5. Kashima K. Comparative study of the palatal rugae and shape of the hard palatal in Japanese and Indian children. Aichi Gakuin Daigaku Shigakkai Shi. 1990;28(1 Pt 2):295-320.

6. Indira A, Gupta M, David M. Usefullness of palatal rugae patterns in establishing identity: preliminary results from Bengaluru city, India. J Forensic Dent Sci. 2012;4(1):2. doi:10.4103/09751475.99149

7. Nayak P, Acharya AB, Padmini AT, Kaveri H. Differences in the palatal rugae shape in two populations of India. Arch Oral Biol. 2007;52(10):977-982. doi:10.1016/j.archoralbio.2007.04.006

8. De Angelis D, Riboli F, Gibelli D, Cappella A, Cattaneo C. Palatal rugae as an individualising marker: reliability for forensic odontology and personal identification. Sci Justice. 2012;52(3):181-184. doi:10.1016/j.scijus.2011.09.002

9. Byatnal A, Byatnal A, Kiran A, Samata Y, Guruprasad Y, Telagi N. Palatoscopy: an adjunct to forensic odontology: a comparative study among five different populations of India. J Nat Sci Biol Med. 2014;5 (1):52-55. doi:10.4103/0976-9668.127287

10. Madhankumar S. Palatal rugae pattern for gender identification among selected student population in Chennai, India. J Sci Res Rep. 2013;2(2):491-496. doi:10.9734/jsrr/2013/4873

11. Pereira T, Shetty S, Surve R, Gotmare S, Kamath P, Kumar S. Palatoscopy and odontometrics for sex identification and hereditary pattern analysis in a Navi Mumbai population: a cross-sectional study. J Oral Maxillofac Pathol. 2018;22(2):271-278. doi:10.4103/ jomfp.JOMFP_197_16

12. Saxena E, Hongal S, Torwane N, Mishra P, Chandrashekhar B, Goel P. A study of the palatal rugae pattern among male female and transgender population of Bhopal city. J Forensic Dent Sci. 2015;7(2):142. doi:10.4103/0975-1475.146370 
13. Sherif AF, Hashim AA, Al Hanafy MA, Soliman EM. A pilot- cross sectional study of palatal rugae shape and direction among Egyptians and Malaysians. Egypt J Forensic Sci. 2018;8(1). doi:10.1186/ s41935-018-0050-1

14. Azab SMS, Magdy R, Sharaf El Deen MA. Patterns of palatal rugae in the adult Egyptian population. Egypt J Forensic Sci. 2016;6 (2):78-83. doi:10.1016/j.ejfs.2015.01.006

15. Gondivkar SM, Patel S, Gadbail AR, Gaikwad RN, Chole R, Parikh RV. Morphological study of the palatal rugae in western Indian population. $J$ Forensic Leg Med. 2011;18(7):310-312. doi:10.1016/j.jflm.2011.06.007

16. Gaikwad R, Kamble S, Rana R, Jain S, Gondivkar S, Bajad P. Rugae patterns as an adjunct to sex differentiation in forensic identification. Stomatologija. 2019;21(3):79-82.

17. Almunajem YS, Almoshaiti YK, Alharbi YR, Alanezi FF, Gaikwad R, Alam MK. Morphological study of the palatal rugae. J Crit Rev. 2020;7(18):2966-2970. doi:10.31838/jcr.07.18.367

18. Pakshir F, Ajami S, Pakshir HR, Malekzadeh AR. Characteristics of palatal rugae patterns as a potential tool for sex discrimination in a sample of Iranian children. J Dent. 2019;20(1):1-9. doi:10.30476/ dentjods.2019.44556

19. Thomas CJ, Kotze TJ. The palatal ruga pattern in six southern African human populations. Part I: a description of the populations an a method for its investigation. J Dent Assoc $S$ Afr. 1983;38 (3):158-165
20. EI-Sharkawy G. A study of palatal rugae pattern (Rugoscopy) in an Egyptian population. Egypt Dent J. 1998;44(3).

21. Hauser G, Daponte A, Roberts MJ. Palatal rugae. $J$ Anat. 1989;165:237-249.

22. Shetty SK, Kalia S, Patil K, Mahima VG. Palatal rugae pattern in Mysorean and Tibetan populations. Indian J Dent Res. 2005;16 (2):51-55.

23. Saraf A, Bedia S, Indurkar A, Degwekar S, Bhowate R. Rugae patterns as an adjunct to sex differentiation in forensic identification. J Forensic Odontostomatol. 2011;29(1):14-19.

24. Dwivedi N, Nagarajappa A. Morphological analysis of palatal rugae pattern in central Indian population. J Int Soc Prev Community Dent. 2016;6(5):417. doi:10.4103/2231-0762.192947

25. Nallamilli S, Tatapudi R, Reddy S, Chennoju S, Kotha R, Kotha P. Diversity of palatal rugae patterns and their reliability in sex discrimination in a South Indian population. J Indian Acad Oral Med Radiol. 2015;27(1):9. doi:10.4103/0972-1363.167066

26. Jibi PM, Gautam KK, Basappa N, Raju OS. Morphological pattern of palatal rugae in children of Davangere. J Forensic Sci. 2011;56 (5):1192-1197. doi:10.1111/j.1556-4029.2011.01831.x

27. Gautam N, Patil SG, Gopi Krishna R, Agastya H, Mushtaq L, Vijay kumar K. Association of palatal rugae pattern in gender identification: an exploratory study. J Contemp Dent Pract. 2017;18 (6):470-473. doi:10.5005/jp-journals-10024-2067

\section{Publish your work in this journal}

Clinical, Cosmetic and Investigational Dentistry is an international, peer-reviewed, open access, online journal focusing on the latest clinical and experimental research in dentistry with specific emphasis on cosmetic interventions. Innovative developments in dental materials, techniques and devices that improve outcomes and patient satisfaction and preference will be highlighted. The manuscript management system is completely online and includes a very quick and fair peer-review system, which is all easy to use. Visit http://www.dovepress.com/testimonials.php to read real quotes from published authors. 